\title{
Some properties of a sequence whose limit is the generalized Ioachimescu's constant
}

\author{
XHEVAT Z. KRASNIQI
}

$\begin{array}{lll}\text { Date of Receiving } & : & 27.02 .2017 \\ \text { Date of Revision } & : & 27.03 .2017 \\ \text { Date of Acceptance } & : & 28.03 .2017\end{array}$

\begin{abstract}
In this short note we have shown some properties of a sequence whose limit is the generalized Ioachimescu's constant. More precise, we show its decreasing monotonicity, concavity, log-concavity, and starshapedness. Our results are simple to be proved but new and with interest. To achieve this we have used on line version of computational knowledge engine Wolfram Alpha.
\end{abstract}

In $1895 \mathrm{~A}$. G. Ioachimescu [1] proposed a problem regarding to the sequence $\left(S_{n}\right)_{n=1}^{\infty}$ defined by

$$
S_{n}=1+\frac{1}{\sqrt{2}}+\cdots+\frac{1}{\sqrt{n}}-2 \sqrt{n}, \quad n \in\{1,2, \ldots\},
$$

who asked to be shown that it is convergent and its limit lies between -2 and -1 .

In [2], the author considered the following modification of above sequence, denoted by $\left(I_{n}\right)_{n=1}^{\infty}$,

$$
I_{n}=1+\frac{1}{\sqrt{2}}+\cdots+\frac{1}{\sqrt{n}}-2(\sqrt{n}-1), \quad n \in\{1,2, \ldots\} .
$$

She has denoted its limit by $\mathcal{J}$ and called it Ioachimescu's constant. Moreover, the same author [3], proved that

$$
\frac{1}{2 \sqrt{n+\frac{1}{5}}}<I_{n}-\mathcal{J}<\frac{1}{2 \sqrt{n+\frac{1}{6}}}, \quad n \in\{1,2, \ldots\} .
$$

Using these inequalities she got that $\mathcal{J}=0.53964549119 \ldots$ In addtion, let us also mention here that the following inequalities pertaining to the sequences $\left(S_{n}\right)_{n=1}^{\infty}$ and $\left(I_{n}\right)_{n=1}^{\infty}$ are given in [5]:

2010 Mathematics Subject Classification. 11Y60, 40A05.

Key words and phrases. sequence, convergence, Ioachimescu's constant, concavity, starshaped sequence.

We are grateful to the referee for her/his constructive input. Her/his comments and suggestions definitely have improved our paper.

Communicated by: I. Milovanovic 